\title{
WELL-BALANCED SCHEMES FOR CONSERVATION LAWS WITH SOURCE TERMS BASED ON A LOCAL DISCONTINUOUS FLUX FORMULATION
}

\author{
KENNETH HVISTENDAHL KARLSEN, SIDDHARTHA MISHRA, \\ AND NILS HENRIK RISEBRO
}

\begin{abstract}
We propose and analyze a finite volume scheme of the Godunov type for conservation laws with source terms that preserve discrete steady states. The scheme works in the resonant regime as well as for problems with discontinuous flux. Moreover, an additional modification of the scheme is not required to resolve transients, and solutions of nonlinear algebraic equations are not involved. Our well-balanced scheme is based on modifying the flux function locally to account for the source term and to use a numerical scheme especially designed for conservation laws with discontinuous flux. Due to the difficulty of obtaining $B V$ estimates, we use the compensated compactness method to prove that the scheme converges to the unique entropy solution as the discretization parameter tends to zero. We include numerical experiments in order to show the features of the scheme and how it compares with a wellbalanced scheme from the literature.
\end{abstract}

\section{INTRODUCTION}

In this paper we study conservation laws with source terms, often referred to as balance laws, a prototype of which is given by

$$
\begin{cases}u_{t}+f(u)_{x}=A(x, u), & (x, t) \in \mathbb{R} \times \mathbb{R}_{+}, \\ u(x, 0)=u_{0}(x), & x \in \mathbb{R},\end{cases}
$$

where $u$ is the (scalar) unknown, $f$ is the flux function, and $A$ is the source term. Frequently the source term takes the form

$$
A(x, u)=z^{\prime}(x) b(u),
$$

in which case (1.1) can be seen as a model equation for the Saint-Venant (shallow water) equations. We remark that the coefficient $z$ in (1.2) can be discontinuous, which would correspond to a discontinuous bottom topography.

Formally (1.1) with the source (1.2) is equivalent to

$$
U_{t}+A U_{x}=0,
$$

Received by the editor November 9, 2006 and, in revised form, October 10, 2007.

2000 Mathematics Subject Classification. Primary 35L65, 74S10, 65M12.

Key words and phrases. Conservation law, discontinuous solution, source term, finite volume scheme, well-balanced scheme, convergence, compensated compactness method.

The third author was supported in part by an Outstanding Young Investigators Award from the Research Council of Norway. 
where $U=(u, z)$ and the matrix $A$ is given by

$$
A=\left(\begin{array}{cc}
f^{\prime}(u) & -b \\
0 & 0
\end{array}\right)
$$

The eigenvalues of the above matrix (wave speeds) are $f^{\prime}(u)$ and 0 , which can coincide and thereby result in "resonance".

If $z(x)=x$, then (1.2) reduces to

$$
u_{t}+(f(u))_{x}=b(u),
$$

which is the usual case of an autonomous source term. If $b(u) \equiv 1$, (1.2) reduces to

$$
u_{t}+(f(u))_{x}=z^{\prime}(x) .
$$

In fact, (1.3) can be written in the following conservative form,

$$
u_{t}+(f(u)-z(x))_{x}=0
$$

which is an example of a conservation law with a spatially varying coefficient. These equations, (1.4) in particular, when the coefficients are discontinuous, have been studied from a theoretical and numerical point of view in a large number of papers; cf. 1, 2, 4, 10, 14, 18, 21, 27, 28, and the references therein. The link between (1.1) and (1.4) will be the basis for the numerical scheme introduced in this paper.

Solutions of (1.1) must be interpreted in the weak sense, and so-called entropy conditions are used to select a unique weak solution to the initial-value problem. This solution is referred to as an entropy solution. Weak and entropy solutions of (1.2) are well defined when $z^{\prime} \in L^{\infty}$ (cf. Section 2) . Whenever $b$ is independent of $u$, one can interpret (1.4) in the sense of distributions, even for discontinuous $z$.

One of the key issues in designing numerical schemes for (1.1) is the resolution of steady states. For the continuous problem, at a steady state $\bar{u}=\bar{u}(x)$ the flux function $f$ and the source term $A$ are balanced, i.e., $\bar{u}$ satisfies

$$
f(u)_{x}=A(x, u) .
$$

More detailed forms of (1.5) can be derived for (1.2) (cf. Section 2). The usual strategy of devising numerical methods for (1.1) is to use a Godunov type numerical flux in a finite volume method coupled with a centered differencing of the source term. It is well known that this does not preserve discrete steady states 13 . Another alternative is provided by the so-called splitting or fractional steps method, which is based on separating the updates for the flux and the source [19]. This method is also deficient with regard to preserving discrete steady states.

Because of these difficulties, so-called well-balanced schemes have been proposed. These schemes are designed specifically for preserving steady states. A variety of well-balanced schemes can be found in literature; see [13, 11, 5, 6] and the references cited therein. For a partial overview, see also the introductory part of [15].

Our aim in this paper is to devise a well-balanced scheme for (1.1). The key element of our strategy will be a "local" transformation of the balance law (1.1) to a conservation law with a space-time dependent discontinuous coefficient:

$$
u_{t}+\tilde{f}(k(x, t), u)_{x}=0
$$

where $\tilde{f}$ is the flux modified locally by the source. Equations of this type are by now mathematically well-studied within a proper framework of entropy solutions, and various types of numerical methods have been devised and analyzed for these equations (cf. the list of references given above and for (1.6) in particular reference 
[16]). Thus, our strategy is to employ numerical schemes designed for conservation laws with discontinuous coefficients (1.6) to approximate solutions of (1.1) (details are presented in Section 3). This strategy is similar in spirit to that used in [5, 19].

The finite volume scheme devised herein is designed to preserve discrete steady states and can therefore be called well-balanced. The scheme is very simple to implement and does not require solving algebraic equations or an addtional entropy treatment for discontinuous steady states. It resolves the transients to first order and the steady states are resolved to machine precision. The main features of the scheme are demonstrated through a series of numerical experiments in Section 5. We believe that the approach of using a local discontinuous flux formulation for designing well-balanced schemes will lead to alternative numerical schemes for systems of conservation laws as well, including the shallow water equations with bottom topography and the Euler equations for flow in a nozzle. We plan to address the extension to systems in future works.

Regarding convergence analysis of well-balanced schemes, if $f^{\prime} \neq 0$, it is possible to work within the standard $B V$ (bounded variation) framework; see, e.g., 11, 12. When resonance occurs, i.e., if $f^{\prime}(u)=0$ for some $u$, the situation becomes more complicated. As with other problems (equations with discontinuous flux) experiencing resonance phenomena, there is generally no spatial variation bound for the conserved variable $u$ itself. In order to show compactness of approximate solutions, the so-called singular mapping approach has been used in the last twenty years, in particular for conservation laws with discontinuous coefficients; cf. 1, 2, 10, 14, 18, 21, 27, 28. More recently, alternative analytical tools have been utilized for discontinuous flux problems, including compensated compactness [16, 17] and entropy process solutions/kinetic solutions 4]. Regarding convergence analysis for equations with source terms, very few of the papers deal with the resonant case where $B V$ estimates are not available. Indeed, we know only of 24, 6, 14. In 24. 14] the singular mapping technique is used to prove the convergence of the Glimm, Godunov, and front tracking methods, while the authors of [6] prove the convergence of so-called equilibrium schemes using the method of kinetic solutions.

In the present paper we show that under suitable hypotheses on the flux function and the source term, the approximate solutions generated by our well-balanced scheme converge to the entropy solution of (1.2). The convergence proof utilizes the compensated compactness method [25, 26]. Let $u_{\Delta x}$ denote the approximate solution generated by the well-balanced scheme and let $(S, Q)$ be an entropy-entropy flux pair. Then the main step in the compensated compactness method is to prove that the entropy production $S\left(u_{\Delta x}\right)_{t}+Q\left(u_{\Delta x}\right)_{x}$ is compact in $W_{\text {loc }}^{-1,2}$. We remark that the $W_{\mathrm{loc}}^{-1,2}$ compactness analysis is nontrivial; it relies on the properties of the solution of the Riemann problem for a conservation law with discontinuous flux.

We have organized this paper as follows: In Section 2 we state our assumptions and define the notion of solutions to be used later on. In Section 3 we present our well-balanced scheme, while the convergence analysis is given in Section 4 In Section 5 we report on a series of numerical examples and present a comparison between our scheme and a well-balanced scheme found in the literature. 


\section{Preliminaries}

2.1. Hypotheses. In this section we detail the hypotheses on $f, A, u_{0}$ and recall the notion of entropy solutions for conservation laws. We assume that $f$ satisfies the following assumptions:

(A.1) $f \in C_{\mathrm{loc}}^{1}(\mathbb{R})$.

(A.2) $f$ has finitely many points of extrema.

(A.3) $u \mapsto f(u)$ is genuinely nonlinear. More precisely, $f^{\prime \prime}(u) \neq 0$ for a.e. $u \in \mathbb{R}$.

(A.4) There exist finite constants $M, C_{f}$ such that

$$
|u|>M \text { implies }|f(u)|>C_{f} \log (|u|) .
$$

Next we state the assumptions on the source term $A$.

(A.5) We have that $A(x, 0)=0$, or $A(x, u)=z^{\prime}(x)$. The mapping $u \mapsto A(x, u)$ is locally Lipschitz continuous in $u$ for all $x$ with a Lipschitz constant $C_{A}$.

Regarding the initial data, we assume

(A.6) $u_{0}(x) \in L^{\infty}(\mathbb{R})$.

2.2. Definition of solutions. We define weak solutions of (1.2) as follows:

Definition 2.1. Suppose (A.1) and (A.5) hold. A function $u \in L^{\infty}\left(\mathbb{R} \times \mathbb{R}_{+}\right)$is called a weak solution of (1.1) if for all $\varphi \in C_{c}^{\infty}(\mathbb{R} \times[0, \infty))$,

$$
\int_{\mathbb{R}^{+}} \int_{\mathbb{R}} u \varphi_{t}+f(u) \varphi_{x}+A(x, u) \varphi d x d t+\int_{\mathbb{R}} u(x, 0) \varphi(x, 0)=0 .
$$

Next, we consider the special case

(A.7) $A(x, u)=z^{\prime}(x)$ for some function $z \in L^{\infty}(\mathbb{R}) \cap B V(\mathbb{R})$,

in which case the following definition of a weak solution can be used:

Definition 2.2. Suppose (A.7) holds. A function $u \in L^{\infty}\left(\mathbb{R} \times \mathbb{R}_{+}\right)$is called a weak solution of (1.1) if for all $\varphi \in C_{c}^{\infty}(\mathbb{R} \times[0, \infty))$,

$$
\int_{\mathbb{R}^{+}} \int_{\mathbb{R}} u \varphi_{t}+(f(u)-z(x)) \varphi_{x} d x d t+\int_{\mathbb{R}} u(x, 0) \varphi(x, 0) d x=0 .
$$

Observe that this definition is meaningful even when $z$ is discontinuous, which is due to the conservative form of the source term (A.7)

Weak solutions are not uniquely determined by their initial data and have to be supplemented with an entropy condition to achieve uniqueness. An entropy solution of (1.2) is defined as follows:

Definition 2.3. Suppose (A.5) holds. A function $u \in L^{\infty}\left(\mathbb{R} \times \mathbb{R}_{+}\right)$is called an entropy solution of (1.1) if for all nonnegative $\varphi \in C_{c}^{\infty}(\mathbb{R} \times[0, \infty))$ and for all entropy-entropy flux pairs $(S, Q)$ the following inequality holds:

$$
\int_{\mathbb{R}^{+}} \int_{\mathbb{R}} S(u) \varphi_{t}+Q(u) \varphi_{x}+S^{\prime}(u) A(x, u) \varphi d x d t+\int_{\mathbb{R}} S\left(u_{0}(x)\right) \varphi(x, 0) d x \geq 0 .
$$

A pair of $C^{2}$ functions $(S, Q)$ is an entropy-entropy flux pair if $S^{\prime \prime} \geq 0, Q^{\prime}=S^{\prime} f^{\prime}$.

It is sufficient to establish (2.1) for the Kružkov entropy-entropy flux pairs

$$
S_{c}(u):=|u-c|, \quad Q_{c}(u)=\operatorname{sign}(u-c)(f(u)-f(c)), \quad c \in \mathbb{R},
$$

where the sign function $\operatorname{sign}(\cdot)$ satisfies $\operatorname{sign}(0)=0$. It is a well-known fact that there exists a unique entropy solution $u$ of (1.1) if $u_{0} \in L^{\infty}(\mathbb{R})$ and $A(x, u)$ is 
Lipshitz continuous in $x$ and locally Lipschitz continuous in $u$. Moreover, if $u_{0} \in$ $B V(\mathbb{R})$, then $u$ belongs to $B V(\mathbb{R} \times(0, T))$ for any $T>0$.

Let us now turn to the singular case (A.7) For the sake of having a wellposed problem at our disposal, we shall reinforce (A.7) by introducing the following "piecewise smoothness" assumption:

(A.8) Condition (A.7) holds and $z(x)$ is piecewise $C^{1}$ with a finite number of jump discontinuity points located at $x_{0}<x_{1}<\cdots<x_{M}, M \geq 0$.

Under this condition we will employ a notion of an entropy solution taken from [15.

Definition 2.4. Suppose (A.8) holds. A function $u$ is said to be an entropy solution of (1.3) if for all $c \in \mathbb{R}$ and for all $0 \leq \varphi \in C_{c}^{\infty}(\mathbb{R} \times[0, \infty))$,

$$
\begin{aligned}
& \int_{\mathbb{R}} \int_{\mathbb{R}_{+}}|u-c| \varphi_{t}+\operatorname{sign}(u-c)(f(u)-f(c)) \varphi_{x} d x d t \\
& \quad+ \int_{\mathbb{R}_{-D}} \int_{\mathbb{R}_{+}} \operatorname{sign}(u-c) z^{\prime}(x) \varphi d x d t \\
& \quad+\sum_{m=0}^{M} \int_{\mathbb{R}_{+}}\left|z\left(x_{m}+\right)-z\left(x_{m}-\right)\right| \varphi\left(x_{m}, t\right) d t+\int_{\mathbb{R}}\left|u_{0}-c\right| \varphi d x \geq 0,
\end{aligned}
$$

where $D=\left\{x_{0}, x_{1}, \ldots, x_{M}\right\}$.

For various existence and uniqueness results for entropy solutions in the sense of Definition 2.3 we refer to [14].

2.3. Convergence framework. We will use the compensated compactness method 25. 26] to prove convergence of our approximate solutions. For simplicity, we will use the Young measure independent version of this method [7, 20].

The following lemma, which contains the compensated compactness method as we rely on it herein, is taken from [7, 20].

Lemma 2.1. Let $\left\{u^{\varepsilon}\right\}_{\varepsilon>0}$ be a sequence of functions such that:

(i) $\left|u^{\varepsilon}\right| \leq C$ for all $\varepsilon>0$.

(ii) The two sequences

$$
\left\{S_{1}\left(u^{\varepsilon}\right)_{t}+Q_{1}\left(u^{\varepsilon}\right)_{x}\right\}_{\varepsilon>0} \quad \text { and } \quad\left\{S_{2}\left(u^{\varepsilon}\right)_{t}+Q_{2}\left(u^{\varepsilon}\right)_{x}\right\}_{\varepsilon>0}
$$

are in a compact subset of $W_{\mathrm{loc}}^{-1,2}\left(\mathbb{R} \times \mathbb{R}^{+}\right)$, where

$$
\begin{gathered}
S_{1}(u)=u-c, \quad Q_{1}(u)=f(u)-f(c), \\
S_{2}(u)=f(u)-f(c), \quad Q_{2}(u)=\int_{c}^{u}\left(f_{u}(\xi)\right)^{2} d \xi,
\end{gathered}
$$

for all $c \in \mathbb{R}$.

Then there is a subsequence of $\left\{u^{\varepsilon}\right\}$ converging a.e. to a function $u \in L^{\infty}\left(\mathbb{R} \times \mathbb{R}^{+}\right)$.

We will also need the following technical result [8] (see also [23]).

Lemma 2.2. Let $\Omega \subset \mathbb{R}^{d}$ be an open set. If $1<q<2<r<\infty$, then

$$
\begin{aligned}
& \left\{\text { compact set of } W_{\mathrm{loc}}^{-1, q}(\Omega)\right\} \bigcap\left\{\text { bounded set of } W_{\mathrm{loc}}^{-1, r}(\Omega)\right\} \\
& \subset\left\{\text { compact set of } W_{\mathrm{loc}}^{-1,2}(\Omega)\right\} .
\end{aligned}
$$




\section{The numerical SCHEME}

In this section we define our the well-balanced scheme for (1.1) and (1.2), which is a Godunov type finite volume scheme.

Let $\Delta t$ and $\Delta x$ be the time step and mesh size respectively. Fix $T>0$ and set

$$
C_{u}=M \exp (C T),
$$

where $C$ is a constant to be determined later. Now set

$$
M_{f}=\max _{\left[-C_{u}, C_{u}\right]}\left|f^{\prime}(u)\right| \text {. }
$$

We assume that the time step and the mesh size satisfy the following CFL-condition:

$$
2 \lambda M_{f} \leq 1, \quad \lambda=\frac{\Delta t}{\Delta x} .
$$

Let $t^{n}=n \Delta t$, and $x_{j}=j \Delta x$ for $n=0,1,2, \ldots$ and $j=\ldots,-1,-1 / 2,0,1 / 2,1, \ldots$ Let $I_{j}$ denote the interval $\left[x_{j-1 / 2}, x_{j+1 / 2}\right)$ and $I^{n}$ the interval $\left[t^{n}, t^{n+1}\right)$. Set

$$
\mathbf{1}_{j}^{n}(x, t)=\mathbf{1}_{I_{j}}(x) \mathbf{1}_{I^{n}}(t),
$$

where $\mathbf{1}_{\Omega}$ denotes the characteristic function of the set $\Omega$.

The initial data is defined by

$$
u_{j}^{0}=\frac{1}{\Delta x} \int_{x_{j-1 / 2}}^{x_{j+1 / 2}} u(x, 0) d x .
$$

Fixing a time level $t^{n}$ on which our approximate solution $u_{j}^{n}$ is given, we describe next how to construct the approximate solution $u_{j}^{n+1}$ at the subsequent time level $t^{n+1}$. Let $u^{n}(x)$ and $B^{n}(x)$ be defined as

$$
u^{n}(x)=\sum_{j} u_{j}^{n} \mathbf{1}_{I_{j}}(x), \quad B^{n}(x)=\sum_{j} B_{j}^{n} \mathbf{1}_{I_{j}}(x)
$$

and

$$
B_{j}^{n}-B_{j-1}^{n}=q_{\Delta x}\left(x_{j-1}, x_{j}, A\left(x, u^{n}(x)\right)\right)
$$

where $q_{\Delta x}$ is some approximate integration such that

$$
q_{\Delta x}(a, b, h(x)) \rightarrow \int_{a}^{b} h(x) d x, \quad \text { as } \Delta x \rightarrow 0,
$$

for bounded functions $h$. In order to determine $B$ completely, we also need to give a starting boundary value $B_{j_{0}}^{n}$ to start the above iteration. We simply choose $B_{j_{0}}^{n}$ to be zero in the numerical experiments presented in this paper. The choice of the starting value is not at all important in the scheme and several choices of the starting value led to the same numerical solutions. For $t \in\left[t^{n}, t^{n+1}\right)$ we solve the following conservation law with a discontinuous coefficient:

$$
u_{t}+\left(f(u)-B^{n}(x)\right)_{x}=0, \quad u\left(x, t^{n}\right)=u^{n}(x),
$$

and then define $u_{j}^{n+1}$ as

$$
u_{j}^{n+1}=\frac{1}{\Delta x} \int_{I_{j}} u\left(y, t^{n+1}\right) d y .
$$

By the CFL-condition, waves emerging from the Riemann problems at $x=x_{j+1 / 2}$ will not interact, and therefore we obtain

$$
u_{j}^{n+1}=u_{j}^{n}-\lambda\left(F_{j+1 / 2}-F_{j-1 / 2}\right),
$$

where $F_{j+1 / 2}$ is the flux across $x=x_{j+1 / 2}$. 
The flux $F_{j+1 / 2}$ is determined by solving the Riemann problem for a conservation law with discontinuous flux:

$$
\begin{cases}u_{t}+\left(f(u)-B_{j}^{n}\right)_{x}=0, \quad u(x, 0)=u_{j}^{n}, & x<x_{j+1 / 2}, \\ u_{t}+\left(f(u)-B_{j+1}^{n}\right)_{x}=0, \quad u(x, 0)=u_{j+1}^{n}, & x \geq x_{j+1 / 2} .\end{cases}
$$

The Riemann problems (3.3) can be exactly solved [10, 3, 22. These formulas provide simple expressions for the Godunov flux to be used across each interface. For example, if $f$ has only one minimum for $u=\theta$ and no maxima, then we have the following formula for the interface flux:

$$
F_{j+1 / 2}=\max \left\{f\left(\max \left\{u_{j}^{n}, \theta\right\}\right)+B_{j}^{n}, f\left(\min \left\{\theta, u_{j+1}^{n}\right\}\right)+B_{j+1}^{n}\right\} .
$$

For more general flux functions, similar explicit formulas have been obtained in 3 . We omit them here for brevity.

For the convergence analysis, we define an approximate solution $u_{\Delta x}$ for $t \neq t^{n}$ and $x \in \mathbb{R}$ by setting

$$
u_{\Delta x}(x, t)=u(x, t) \quad t^{n} \leq t<t^{n+1}, x \in \mathbb{R},
$$

where $u$ is the entropy solution of (3.1); cf. Definition 2.4

We are going to show in the subsequent section that (3.2) is a well-balanced scheme (preserves discrete steady states exactly) and that $u_{\Delta x}$ converges as $\Delta x \rightarrow 0$ to an entropy solution of (1.2).

\section{Convergence analysis}

In this section, we will carry out the convergence analysis for the well-balanced Godunov type scheme (3.2). First, we show that the scheme preserves discrete steady states. A discrete steady state for our scheme is defined by

$$
f\left(u_{j+1}^{n}\right)-f\left(u_{j}^{n}\right)=B_{j+1}^{n}-B_{j}^{n}, \quad j \in \mathbb{Z},
$$

where we observe that

$$
B_{j+1}^{n}-B_{j}^{n} \approx \int_{x_{j}}^{x_{j+1}} A\left(y, u^{n}(y)\right) d y
$$

This is the flux-source balance that characterizes steady states. In the special case of (1.2) we use the formula,

$$
\begin{aligned}
B_{j+1}^{n}-B_{j}^{n}=(z & \left.\left(x_{j+1 / 2}-\right)-z\left(x_{j}+\right)\right) b\left(u_{j}^{n}\right) \\
& +\left(z\left(x_{j+1}-\right)-z\left(x_{j+1 / 2}+\right)\right) b\left(u_{j+1}^{n}\right) .
\end{aligned}
$$

The well-balancing properties of the scheme are defined below.

Lemma 4.1. Let $\left\{u_{j}^{n}\right\}_{j \in \mathbb{Z}}$ be a sequence such that

$$
f\left(u_{j+1}^{n}\right)-f\left(u_{j}^{n}\right)=B_{j+1}^{n}-B_{j}^{n}, \quad \forall j \in \mathbb{Z},
$$

and

$$
f^{\prime}\left(u_{j}^{n}\right) f^{\prime}\left(u_{j+1}^{n}\right)<0 \Longrightarrow f^{\prime}\left(u_{j}^{n}\right)>0 \quad \text { and } \quad f^{\prime}\left(u_{j+1}^{n}\right)<0, \quad \forall j \in \mathbb{Z} .
$$

Then

$$
u_{j}^{n+1}=u_{j}^{n}, \quad \forall j \in \mathbb{Z},
$$

where $\left\{u_{j}^{n+1}\right\}_{j \in \mathbb{Z}}$ is computed by the scheme (3.2). 
Proof. Since $u_{j}^{n}$ satisfies both the Rankine-Hugoniot condition and the entropy condition (4.2) across each interface $x_{j+1 / 2}$, we have that

$$
F_{j+1 / 2}=f\left(u_{j}^{n}\right)+B_{j}^{n}=f\left(u_{j+1}^{n}\right)+B_{j+1}^{n}, \quad \forall j \in \mathbb{Z} .
$$

Therefore, $F_{j+1 / 2}=F_{j-1 / 2}$ and the lemma is proved.

Remark 4.1. Lemma 4.1 says that a discrete steady state (4.1) satisfying condition (4.2) is preserved by the well-balanced scheme (3.2). The additional constraint (4.2) is an entropy condition essentially excluding under-compressive waves.

Remark 4.2. If the steady states of (1.2) are smooth and, in addition, we assume that $z$ is also smooth, then we have a more explicit representation of the flux-source balance. If $b(u) \neq 0$, we can formally write (1.5) as

$$
D(u)-z(x)=C, \quad D(u)=\int_{0}^{u} \frac{f^{\prime}(s)}{b(s)} d s,
$$

for some constant $C$. It is clear that (4.3) constitutes a nonlinear algebraic equation from which a steady state can be calculated.

It is easy to check that the discrete steady state (4.1) differs from the steady state (4.3) by order of the truncation error. This is consistent with any discrete form of (4.3) which may also differ from the continuous steady state by the order of the truncation error. We have chosen to preserve the discrete steady state (4.1) as it is more general, and the flux-source balance (1.5) is valid (in the weak form) when the steady states and the function $z$ are no longer smooth. It is also valid for the more general case of (1.1) where a simple algebraic formula (4.3) is no longer available.

Remark 4.3. Note that we have some flexibility in the choice of $B$ in (4.1) as it is based on a quadrature of $A$. Two different choices for $B$ will lead to different discrete steady states. When $A$ is continuous, these two discrete steady states (corresponding to different $B$ 's) differ by the order of the truncation error.

Next, we will show that the approximate solutions are bounded in $L^{\infty}$.

Lemma 4.2. There exists a constant $\sigma$, independent of $\Delta x$, such that

$$
\left|u_{\Delta x}\left(x, t^{n}\right)\right| \leq M e^{\sigma t^{n}}, \quad M:=\left\|u_{0}\right\|_{L^{\infty}(\mathbb{R})} .
$$

Proof. Let $M, C_{A}, C_{f}$ be the constants defined in (A.4) and (A.5). Without loss of generality, we can assume that $u_{j}^{0}<M$. Now either $f(u)>C_{f} \log (u)$ or $f(u)<$ $-C_{f} \log (u)$ for $u>M$. We assume that $f(u)>C_{f} \log (u)$, the other case being similar. To start we assume inductively that $u_{j}^{n}<M e^{\sigma t^{n}}=: M_{n}$. Any Riemann problem arising at $t=t^{n}$ will involve left and right flux functions whose difference is bounded by

$$
\Delta x C_{A} \leq \lambda^{-1} \Delta t C_{A}=: \Delta .
$$

Furthermore, any left and right states are smaller than $M_{n}$. Therefore, any states in the solution will be less than $\bar{u}$, where $\bar{u}$ solves

$$
f(\bar{u})=f\left(M_{n}\right)+\Delta .
$$

Therefore,

$$
C_{f}\left(\log (\bar{u})-\log \left(M_{n}\right)\right) \leq \Delta
$$

or

$$
\bar{u} \leq M_{n} e^{\Delta / C_{f}}=M e^{\sigma t^{n}+C_{A} /\left(\lambda C_{f}\right) \Delta t} .
$$


Setting $\sigma=C_{A} /\left(\lambda C_{f}\right)$ shows that $u_{j}^{n+1}<M e^{\sigma t^{n+1}}$. Showing the corresponding lower bounds is similar.

We will apply the compensated compactness Lemma 2.1 to the sequence $\left\{u_{\Delta x}\right\}$. To this end, we will need to establish certain entropy dissipation estimates to be able to verify the $W_{\text {loc }}^{-1,2}$ compactness of the entropy production associated with $\left\{u_{\Delta x}\right\}$. To prove the dissipation estimates we will adapt an approach from [17. developed for conservation laws with discontinuous coefficients.

As $u_{\Delta x}$ locally is the solution of the Riemann problems for conservation laws with discontinuous coefficients, we start by recalling some results relating to the problem

$$
\left\{\begin{array}{l}
u_{t}+g\left(k_{l}, u\right)_{x}=0, \quad u(x, 0)=u_{l} \quad x<0 \\
u_{t}+g\left(k_{r}, u\right)_{x}=0, \quad u(x, 0)=u_{r} \quad x>0
\end{array}\right.
$$

where $k_{l, r}$ and $u_{l, r}$ are given constants. For the moment, we just assume that $g$ is a continuously differentiable function. The Rankine-Hugoniot condition tells us that the values

$$
u_{l, r}^{\prime}=\lim _{x \rightarrow 0 \mp} u(x, t)
$$

satisfy

$$
g_{0}:=g\left(k_{l}, u_{l}^{\prime}\right)=g\left(k_{r}, u_{r}^{\prime}\right) .
$$

In general, this does not determine $u_{l, r}^{\prime}$ uniquely, and we need an additional condition. We use here the so-called minimal jump entropy condition, which states that among the possible choices we select $u_{l}^{\prime}$ and $u_{r}^{\prime}$ such that $\left|u_{l}^{\prime}-u_{r}^{\prime}\right|$ is minimal. This choice has the following consequences (see [10]):

$$
\begin{aligned}
& u_{l}^{\prime} \leq u_{r}^{\prime} \Longrightarrow\left\{\begin{array}{lc}
g\left(k_{l}, u\right) \geq g\left(k_{l}, u_{l}^{\prime}\right) & \text { for all } u \in\left[u_{l}^{\prime}, u_{r}^{\prime}\right], \text { or } \\
g\left(k_{r}, u\right) \geq g\left(k_{r}, u_{r}^{\prime}\right) & \text { for all } u \in\left[u_{l}^{\prime}, u_{r}^{\prime}\right],
\end{array}\right. \\
& u_{r}^{\prime} \leq u_{l}^{\prime} \Longrightarrow\left\{\begin{array}{lc}
g\left(k_{l}, u\right) \leq g\left(k_{l}, u_{l}^{\prime}\right) & \text { for all } v \in\left[u_{r}^{\prime}, u_{l}^{\prime}\right], \text { or } \\
g\left(k_{r}, u\right) \leq g\left(k_{r}, u_{r}^{\prime}\right) & \text { for all } u \in\left[u_{r}^{\prime}, u_{l}^{\prime}\right] .
\end{array}\right.
\end{aligned}
$$

Lemma 4.3 ([17]). If the values $u_{l}^{\prime}$ and $u_{r}^{\prime}$ are chosen according to the minimal jump entropy condition, then for any constant $c$,

$$
Q_{r}\left(u_{r}^{\prime}, c\right)-Q_{l}\left(u_{l}^{\prime}, c\right) \leq\left|g\left(k_{r}, c\right)-g\left(k_{l}, c\right)\right|,
$$

where $Q_{l}$ and $Q_{r}$ denote the Kružkov entropy fluxes

$$
\begin{aligned}
& Q_{l}(v, c)=\operatorname{sign}(v-c)\left(g\left(k_{l}, v\right)-g\left(k_{l}, c\right)\right), \\
& Q_{r}(v, c)=\operatorname{sign}(v-c)\left(g\left(k_{r}, v\right)-g\left(k_{r}, c\right)\right) .
\end{aligned}
$$

Next we continue with the proofs of the entropy dissipation estimates. We shall use the notation

$$
\llbracket \alpha \rrbracket(x, t)=\lim _{h \rightarrow 0} \alpha(x+h, t)-\alpha(x-h, t),
$$

for any quantity $\alpha=\alpha(x, t)$. Fix an entropy-entropy flux pair $(S, Q)$. Then the entropy dissipation of $u_{\Delta x}$ associated with $(S, Q)$ is defined to be

$$
E(\varphi)=\iint_{\Pi_{X, T}} S\left(u_{\Delta x}\right) \varphi_{t}+Q\left(u_{\Delta x}\right) \varphi_{x} d x d t, \quad \varphi \in C_{c}^{\infty}\left(\mathbb{R} \times \mathbb{R}_{+}\right),
$$


where we let $\Pi_{X, T}$ denote the set $[-X, X] \times[0, T]$ and where $\varphi \in C^{\infty}\left(\Pi_{X, T}\right)$. Without loss of generality, we can assume that $X$ and $T$ are such that $X=x_{J+1 / 2}$ and $T=t^{N}$ for some integers $J$ and $N$.

By an integration by parts and the local Riemann solution structure of the approximation $u_{\Delta x}$, we can split $E(\varphi)$ as

$$
E(\varphi)=I_{1}(\varphi)+I_{2}(\varphi)+I_{3}(\varphi)+I_{4}(\varphi)+I_{5}(\varphi),
$$

where

$$
\begin{aligned}
& I_{1}(\varphi)=\left.\int_{-X}^{X} S\left(u_{\Delta x}(x, t)\right) \varphi(x, t)\right|_{t=0} ^{t=T} d x, \\
& I_{2}(\varphi)=\sum_{n=0}^{N-1} \int_{-X}^{X}\left[S\left(u_{\Delta x}\left(x, t^{n}-\right)\right)-S\left(u_{\Delta x}\left(x, t^{n}+\right)\right)\right] \varphi\left(x, t^{n}\right) d x, \\
& I_{4}(\varphi)=\sum_{j=-J}^{J} \sum_{n=0}^{N-1} \int_{t^{n}}^{t^{n+1}}\left[Q\left(u_{j+1 / 2}^{n,-}\right)-Q\left(u_{j+1 / 2}^{n,+}\right)\right] \varphi\left(x_{j}, t\right) d t, \\
& I_{5}(\varphi)=\left.\sum_{n=0}^{N-1} \int_{t^{n}}^{t^{n+1}}\left(Q\left(u_{j-1 / 2}^{n}\right) \varphi\left(x_{j}, t\right)\right)\right|_{j=-J} ^{j=J} d t, \\
& I_{3}(\varphi)=\sum_{j=-J}^{J} \sum_{n=0}^{N-1} \sum_{\sigma} \int_{t^{n}}^{t^{n+1}}[\sigma \llbracket S \rrbracket+\llbracket Q \rrbracket] \varphi\left(x_{j+1 / 2}+\sigma t, t\right) d t,
\end{aligned}
$$

where the summation over $\sigma$ extends to all shocks with speeds $\sigma$ in the solution of the Riemann problem at the interface $x_{j+1 / 2}$. We have also used the notation

$$
u_{j+1 / 2}^{n, \pm}=\lim _{x \rightarrow x_{j+1 / 2} \pm} u_{\Delta x}\left(x, t^{n}\right) .
$$

We have the following lemma on the variation across each time level.

Lemma 4.4. Let $u_{\Delta x}$ and $u_{j}^{n}$ be generated by the well-balanced scheme (3.2). There exists a constant $C=C(X, T)$ independent of $\Delta x$ such that

$$
\sum_{n=1}^{N-1} \sum_{j=-J}^{J} \int_{I_{j}}\left(u^{\Delta x}\left(x, t^{n}-\right)-u_{j}^{n}\right)^{2} d x \leq C .
$$

Proof. In what follows we use the entropy-entropy flux pair

$$
S(u)=\frac{1}{2} u^{2}, \quad Q_{u}(u)=u f_{u}(u) .
$$

Without loss of generality, we assume that the numerical solution $u_{\Delta x}$ has compact support, so that we can use the test function $\varphi=1$, which implies $E(\varphi)=0$.

Since $u_{\Delta x}$ is bounded,

$$
\left|I_{1}(\varphi)\right| \leq C_{1}(X, T) \text { and }\left|I_{5}(\varphi)\right| \leq C_{5}(X, T) .
$$


Next we estimate $I_{2}$. Writing $u_{-}^{n}$ for $u_{\Delta x}\left(\cdot, t^{n}-\right)$ we find

$$
\begin{aligned}
I_{2}(\varphi) & =\sum_{n, j} \int_{I_{j}} S\left(u_{-}^{n}\right)-S\left(u_{j}^{n}\right) d x \\
& =\frac{1}{2} \sum_{n, j} \int_{I_{j}}\left(u_{-}^{n}\right)^{2}-\left(u_{j}^{n}\right)^{2} d x \\
& =\frac{1}{2} \sum_{n, j} \int_{I_{j}}\left(u_{-}^{n}-u_{j}^{n}\right)^{2} d x-\sum_{n, j} \int_{I_{j}} u_{j}^{n}\left(u_{-}^{n}-u_{j}^{n}\right) d x \\
& =\frac{1}{2} \sum_{n, j} \int_{I_{j}}\left(u_{-}^{n}-u_{j}^{n}\right)^{2} d x,
\end{aligned}
$$

as the second term in the third line above is zero since

$$
u_{j}^{n}=\frac{1}{\Delta x} \int_{I_{j}} u_{-}^{n}(x) d x .
$$

Regarding the term $I_{3}(\varphi)$, we use the fact that $u_{\Delta x}$ is the exact solution of a Riemann problem. Thus, by the entropy condition,

$$
\sigma \llbracket S\left(u_{\Delta x}\right) \rrbracket+\llbracket Q\left(u_{\Delta x}\right) \rrbracket \geq 0,
$$

and consequently,

$$
I_{3}(\varphi) \geq 0
$$

For any convex $C^{2}$ function $S$, we have using Lemma 4.3 and an approximation argument (see 17 for details)

$$
\begin{aligned}
Q\left(u_{j+1 / 2}^{n,+}\right)-Q\left(u_{j+1 / 2}^{n,-}\right) & \leq\left|B_{j+1}^{n}-B_{j}^{n}\right| \\
& \leq \int_{x_{j}}^{x_{j+1}}\left|A\left(y, u^{n}(y)\right)\right| d y \\
& \leq C_{A} \Delta x,
\end{aligned}
$$

where $C_{A}$ is specified in (A.5). If (A.7) holds, then we can bound the last term by the total variation of $z$ in the interval $I_{j+1 / 2}$. In both cases we have

$$
I_{4}(\varphi) \geq-C,
$$

where $C=C(X, T)$ is a positive constant independent of $\Delta x$. This finishes the proof of the lemma.

Estimate (4.7) can be converted into an estimate of the variation of the approximate solutions, which is the content of the subsequent lemma.

Lemma 4.5. Let $u_{j}^{n}$ be defined by the well-balanced scheme (3.2). There exists a constant $C=C(X, T)$ independent of $\Delta x$ such that

$$
\Delta x \sum_{n=0}^{N-1} \sum_{j=-J}^{J}\left(u_{j+1 / 2}^{n,-}-u_{j+1 / 2}^{n,+}\right)^{2}+\left(u_{j}^{n}-u_{j+1 / 2}^{n,-}\right)^{2}+\left(u_{j+1}^{n}-u_{j+1 / 2}^{n,+}\right)^{2} \leq C,
$$

where $J$ and $N$ are such that $T=t^{N}$ and $x_{J+1 / 2}=X$.

Proof. Equipped with (4.7), the proof follows along the lines of 9] (see estimate (7.13) on page 67 in that paper). 
Now we prove the $W_{\text {loc }}^{-1,2}$ compactness of the entropy production associated with the approximate solutions.

Lemma 4.6. Let $u_{\Delta x}$ be generated by the well-balanced scheme (3.2) and let $\left(S_{i}, Q_{i}\right), i=1,2$, be the entropy-entropy flux pairs defined in Lemma 2.1. Define the functional $\hat{E}$ by

$$
\hat{E}(\varphi)=\iint_{\Pi_{X, T}} S_{i}\left(u_{\Delta x}\right) \varphi_{t}+Q_{i}\left(u_{\Delta x}\right) \varphi_{x} d x d t, \quad i=1,2 .
$$

Then the sequence $\{\hat{E}\}_{\Delta x>0}$ is compact in $W_{\mathrm{loc}}^{-1,2}\left(\mathbb{R} \times \mathbb{R}^{+}\right)$.

Proof. First we note that by the $L^{\infty}$ bounds on $u_{\Delta x}$, we have that

$$
|\hat{E}(\varphi)| \leq C\|\varphi\|_{W^{1, \infty}\left(\Pi_{X, T}\right)}
$$

so that $\hat{E}$ is bounded in $W^{-1, r}$ for any $r \in(2, \infty]$. By using the bounds (4.8) and Lemma 4.4 we conclude

$$
\left|I_{1}(\varphi)\right|\left|I_{5}(\varphi)\right|\left|I_{3}(\varphi)\right| \leq C_{1}\|\varphi\|_{L^{\infty}\left(\Pi_{X, T}\right)} .
$$

To estimate the $I_{2}$-term we split it as follows:

$$
I_{2}(\varphi)=I_{2,1}(\varphi)+I_{2,2}(\varphi)
$$

where

$$
\begin{aligned}
& I_{2,1}(\varphi)=\sum_{n, j} \int_{I_{j}}\left(S\left(u_{-}^{n}\right)-S\left(u_{j}^{n}\right)\right) \varphi_{j}^{n} d x \\
& I_{2,2}(\varphi)=\sum_{n, j} \int_{I_{j}}\left(S\left(u_{-}^{n}\right)-S\left(u_{j}^{n}\right)\right)\left(\varphi_{j}^{n}-\varphi\left(x, t^{n}\right)\right) d x,
\end{aligned}
$$

where $\varphi_{j}^{n}=\varphi\left(x_{j}, t^{n}\right)$. Next

$$
S\left(u_{-}^{n}\right)-S\left(u_{j}^{n}\right)=S_{u}\left(u_{j}^{n}\right)\left(u_{-}^{n}-u_{j}^{n}\right)+\frac{1}{2} S_{u u}\left(\theta_{j}^{n}\right)\left(u_{-}^{n}-u_{j}^{n}\right)^{2}
$$

for some intermediate value $\theta_{j}^{n}(x)$. The integral of the first term above over the interval $\left(x_{j-1 / 2}, x_{j+1 / 2}\right)$ is zero. Therefore, we can write

$$
\begin{aligned}
\left|I_{2,1}(\varphi)\right| & =\frac{1}{2}\left|\sum_{n, j} \varphi_{j}^{n} \int_{I_{j}} S_{u u}\left(\theta_{j}^{n}\right)\left(u_{-}^{n}-u_{j}^{n}\right)^{2} d x\right| \\
& \leq C\|\varphi\|_{L^{\infty}\left(\Pi_{X, T}\right)},
\end{aligned}
$$

by Lemma 4.4. Let each term in $I_{2,2}$ be denoted by $I_{2,2}^{j, n}$. Then

$$
\begin{aligned}
\left|I_{2,2}^{j, n}(\varphi)\right| & =\left|\int_{I_{j}}\left(S\left(u_{-}^{n}\right)-S\left(u_{j}^{n}\right)\right)\left(\varphi_{j}^{n}-\varphi\left(x, t^{n}\right)\right) d x\right| \\
& \leq\|\varphi\|_{C^{0, \alpha}\left(\Pi_{X, T}\right)} \Delta x^{\alpha} \int_{I_{j}}\left|S\left(u_{-}^{n}\right)-S\left(u_{j}^{n}\right)\right| d x
\end{aligned}
$$

where $\alpha \in(0,1)$ will be chosen later. Then, by a weighted Young's inequality,

$$
\begin{aligned}
\left|I_{2,2}^{j, n}(\varphi)\right| \leq \| & \varphi \|_{C^{0, \alpha}\left(\Pi_{X, T}\right)} \Delta x^{\alpha} \frac{\Delta x^{2 \alpha+1}}{\Delta x^{\delta}} \\
& +\|\varphi\|_{C^{0, \alpha}\left(\Pi_{X, T}\right)} \int_{I_{j}} \Delta x^{\delta}\left(S\left(u_{-}^{n}\right)-S\left(u_{j}^{n}\right)\right)^{2} d x
\end{aligned}
$$


Now, summing over $j, n$, and using Lemma 4.4 as when bounding $I_{2,1}$ we arrive at

$$
\begin{aligned}
\left|I_{2,2}(\varphi)\right| & \leq\|\varphi\|_{C^{0, \alpha}\left(\Pi_{X, T}\right)}\left(\Delta x^{2 \alpha-1-\delta}+C \Delta x^{\delta}\right) \\
& \leq C\|\varphi\|_{C^{0, \alpha}\left(\Pi_{X, T}\right)} \Delta x^{\delta}\left(\Delta x^{2(\alpha-\delta)-1}+1\right) .
\end{aligned}
$$

Therefore, if

$$
\alpha>\frac{1}{2}+\delta
$$

we have that

$$
\left|I_{2,2}(\varphi)\right| \leq C\|\varphi\|_{C^{0, \alpha}\left(\Pi_{X, T}\right)} \Delta x^{\delta} .
$$

Next we estimate the term $I_{4}$ for the entropy-entropy flux pairs defined in (2.4). We have, by the properties of the entropy solution of the Riemann problem for conservation laws with discontinuous flux,

$$
\begin{aligned}
\left|\llbracket Q_{1} \rrbracket_{j+1 / 2}^{n}\right| & =\left|f\left(u_{j+1 / 2}^{n,-}\right)-f\left(u_{j+1 / 2}^{n,+}\right)\right| \\
& =\left|f\left(u_{j+1 / 2}^{n,-}\right)+B_{j}^{n}-f\left(u_{j+1 / 2}^{n,+}\right)-B_{j+1}^{n}+B_{j+1}^{n}-B_{j}^{n}\right| \\
& =\left|B_{j+1}^{n}-B_{j}^{n}\right| \leq \int_{x_{j}}^{x_{j+1}}\left|A\left(y, u^{n}(y)\right)\right| d y \leq C_{A} \Delta x,
\end{aligned}
$$

from which the desired estimate follows:

$$
\left|I_{4}(\varphi)\right| \leq \Delta t \sum_{j, n}\left|\llbracket Q_{1} \rrbracket_{j+1 / 2}^{n}\right| \leq C\|\varphi\|_{L^{\infty}} .
$$

Next we consider the entropy flux $Q_{2}(u)$. We have

$$
\llbracket Q_{2} \rrbracket_{j+1 / 2}^{n}=\int_{u_{j+1 / 2}^{n,+}}^{u_{j+1 / 2}^{n,-}}\left(f_{u}(\xi)\right)^{2} d \xi
$$

By using the $L^{\infty}$ bounds on $u_{\Delta x}$ and the fact that $\left|f_{u}(u)\right| \leq C$ for bounded $u$ we see that

$$
\left|\llbracket Q_{2} \rrbracket_{j+1 / 2}^{n}\right| \leq \int_{u^{-}}^{u^{+}}\left|f_{u}(\xi)\right| d \xi=: q_{j+1 / 2}^{n},
$$

where we have introduced the notation

$$
u^{-}=\min \left\{u_{j+1 / 2}^{n,-}, u_{j+1 / 2}^{n,+}\right\} \quad u^{+}=\max \left\{u_{j+1 / 2}^{n,-}, u_{j+1 / 2}^{n,+}\right\} .
$$

If $f_{u}$ does not change sign in the interval $\left(u^{-}, u^{+}\right)$, we can estimate the jump in $Q_{2}$ as we estimated the jump in $Q_{1}$, concluding that

$$
q_{j+1 / 2} \leq \int_{x_{j}}^{x_{j+1}}\left|A\left(y, u^{n}(y)\right)\right| d y \leq C_{A} \Delta x
$$


Let us assume that $u^{-}<\theta<u^{+}$and that $f_{u}(\theta)=0$. Then, by using a Taylor expansion about $\xi=\theta$,

$$
\begin{aligned}
q_{j+1 / 2} & =\int_{u^{-}}^{u^{+}}\left|f_{u}(\xi)\right| d \xi \leq C \int_{u^{-}}^{u^{+}}|\xi-\theta| d \xi \\
& =C\left(\int_{\theta}^{u^{+}}(\xi-\theta) d \xi-\int_{u^{-}}^{\theta}(\xi-\theta) d \xi\right) \\
& \leq \frac{C}{2}\left(\left(u^{+}-\theta\right)^{2}+\left(\theta-u^{-}\right)^{2}\right) \\
& \leq C\left(u^{+}-u^{-}\right)^{2}=C\left(u_{j+1 / 2}^{n,+}-u_{j+1 / 2}^{n,-}\right)^{2} .
\end{aligned}
$$

So by combining this estimate and (4.11) with (4.10) we conclude that

$$
\left|\llbracket Q_{i} \rrbracket_{j+1 / 2}^{n}\right| \leq C\left(\Delta x+\left(u_{j+1 / 2}^{n,+}-u_{j+1 / 2}^{n,-}\right)^{2}\right), \quad i=1,2
$$

and hence

$$
\begin{aligned}
\left|I_{4}(\varphi)\right| & \leq C \Delta t\|\varphi\|_{L^{\infty}\left(\Pi_{X, T}\right)} \sum_{j, n}\left|\llbracket Q_{i} \rrbracket_{j+1 / 2}^{n}\right| \\
& \leq C\|\varphi\|_{L^{\infty}\left(\Pi_{X, T}\right)}\left(T+\Delta x \sum_{j, n}\left(u_{j+1 / 2}^{n,+}-u_{j+1 / 2}^{n,-}\right)^{2}\right) \\
& \leq C(X, T)\|\varphi\|_{L^{\infty}\left(\Pi_{X, T}\right)} .
\end{aligned}
$$

Now we can use standard arguments (see [16, 17]) to conclude that $\{\hat{E}\}_{\Delta x>0}$ is compact in $W_{\text {loc }}^{-1, q}$ for some $1<q<2$. Hence by Lemma 2.2, we obtain the $W_{\text {loc }}^{-1,2}$ compactness of the approximate solutions.

To prove that any limit of $\left\{u_{\Delta x}\right\}_{\Delta x>0}$ is an entropy solution, we shall need the two succeeding lemmas.

Lemma 4.7. Consider the Riemann problem

$$
\left\{\begin{array}{lll}
u_{t}+\left(f(u)+B_{l}\right)_{x}=0, & u(x, 0)=u_{l}, & x<0, \\
u_{t}+\left(f(u)+B_{r}\right)_{x}=0, & u(x, 0)=u_{r}, & x \geq 0,
\end{array}\right.
$$

where $B_{l, r}$ are constants. Let $u^{\mp}$ denote the limits

$$
u^{\mp}=\lim _{x \rightarrow 0 \mp} u(x, t) .
$$

For each fixed $c \in \mathbb{R}$,

$$
Q_{c}\left(u^{-}\right)-Q_{c}\left(u^{+}\right) \geq \operatorname{sign}(\tilde{u}-c)\left(B_{l}-B_{r}\right),
$$

where $\tilde{u}=u^{-}$or $\tilde{u}=u^{+}$and $Q_{c}$ denotes the Kružkov entropy flux; cf. (2.2).

Proof. Assume first that $\operatorname{sign}\left(u^{-}-c\right)=\operatorname{sign}\left(u^{+}-c\right)$. Then

$$
\begin{aligned}
Q_{c}\left(u^{-}\right)-Q_{c}\left(u^{+}\right) & =\operatorname{sign}\left(u^{\mp}-c\right)\left(f\left(u^{-}\right)-f\left(u^{+}\right)\right) \\
& =\operatorname{sign}\left(u^{\mp}-c\right)\left(B_{l}-B_{r}\right) .
\end{aligned}
$$


Hence the lemma holds in this case. Next assume that $\operatorname{sign}\left(u^{-}-c\right) \neq \operatorname{sign}\left(u^{+}-c\right)$. If $u^{+}<u^{-}$, then we have that $u^{+}<c<u^{-}$. By the minimal jump entropy condition, in this case (4.4), either

$$
f(c) \leq f\left(u^{-}\right) \quad \text { or } \quad f(c) \leq f\left(u^{+}\right) .
$$

Assume now that the first of these inequalities hold. Then, using in addition the Rankine-Hugoniot condition,

$$
\begin{aligned}
Q_{c}\left(u^{-}\right)-Q_{c}\left(u^{+}\right) & =f\left(u^{-}\right)-f(c)+f\left(u^{+}\right)-f(c) \\
& \geq f\left(u^{+}\right)-f(c) \\
& =\left(f\left(u^{+}\right)+B_{r}\right)-\left(f(c)+B_{r}\right) \\
& =\left(f\left(u^{-}\right)+B_{l}\right)-\left(f(c)+B_{r}\right) \\
& \geq\left(f(c)+B_{l}\right)-\left(f(c)+B_{r}\right) \\
& =\operatorname{sign}\left(u^{-}-c\right)\left(B_{l}-B_{r}\right) .
\end{aligned}
$$

If $f(c) \leq f\left(u^{+}\right)$, then we find similarly that

$$
\begin{aligned}
Q_{c}\left(u^{-}\right)-Q_{c}\left(u^{+}\right) & \geq f\left(u^{-}\right)-f(c) \\
& \geq f\left(u^{-}\right)-f\left(u^{+}\right) \\
& =\left(f\left(u^{-}\right)+B_{l}\right)-\left(f\left(u^{+}\right)+B_{r}\right)-\left(B_{l}-B_{r}\right) \\
& =\operatorname{sign}\left(u^{+}-c\right)\left(B_{l}-B_{r}\right) .
\end{aligned}
$$

This concludes the case where $u^{+}<c<u^{-}$. The analysis in the case where $u^{-}<c<u^{+}$is similar.

To prove that a limit function of $\left\{u_{\Delta x}\right\}_{\Delta x}$ is an entropy solution, we shall need the following technical lemma.

Lemma 4.8 ([15]). Let $\Omega \subset \mathbb{R}^{d}$ be a bounded open set, $g \in L^{1}(\Omega)$, and suppose that $\left\{g_{\nu}\right\}_{\nu>0}$ is a sequence such that $g_{\nu} \rightarrow g$ a.e. in $\Omega$ as $\nu \rightarrow 0$. Then there exists a set $\Theta$, which is at most countable, such that for any $c \in \mathbb{R} \backslash \Theta$,

$$
\operatorname{sign}\left(g_{\nu}-c\right) \rightarrow \operatorname{sign}(g-c) \quad \text { a.e. in } \Omega \text { as } \nu \rightarrow 0 .
$$

Let $c \in \Theta$ and define $\mathcal{E}_{c}=\{x \in \Omega \mid g(x)=c\}$. There exists sequences $\left\{\bar{c}_{\nu}\right\}$ and $\left\{\underline{c}_{\nu}\right\}$ such that $\bar{c}_{\nu} \downarrow c$ and $\underline{c}_{\nu} \uparrow c$ as $\nu \rightarrow 0$, and $\bar{c}_{\nu}$ and $\underline{c}_{\nu}$ are in $\mathbb{R} \backslash \Theta$, and

$$
\begin{array}{ll}
\operatorname{sign}\left(g(c)-\underline{c}_{\nu}\right) \rightarrow \operatorname{sign}(g(x)-c) & \text { a.e. } x \in \Omega \backslash \mathcal{E}_{c}, \\
\operatorname{sign}\left(g(c)-\bar{c}_{\nu}\right) \rightarrow \operatorname{sign}(g(x)-c) & \text { a.e. } x \in \Omega \backslash \mathcal{E}_{c} .
\end{array}
$$

Now we are in a position to state our main convergence theorem.

Theorem 4.1. Suppose conditions (A.1) (A.6) hold. Let $u_{\Delta x}$ be the approximate solutions generated by the well-balanced Godunov type scheme (3.2). Then there exists a limit function $u \in L^{\infty}\left(\mathbb{R} \times \mathbb{R}_{+}\right)$such that

$$
u_{\Delta x} \stackrel{\Delta x \rightarrow 0}{\longrightarrow} u \quad \text { in } L_{\text {loc }}^{p}\left(\mathbb{R} \times \mathbb{R}_{+}\right) \text {for } p<\infty \text { and a.e. in } \mathbb{R} \times \mathbb{R}_{+} .
$$

Furthermore, $u$ is an entropy solution of (1.2).

Proof. The claimed convergence of $\left\{u_{\Delta x}\right\}_{\Delta x>0}$ to a limit function $u$ is a straightforward consequence of Lemmas 4.6 and 2.1 
Let us now prove that the limit function $u$ is an entropy solution in the sense of Definition 2.4. Fixing any constant $c \in \mathbb{R}$, we consider in what follows the Kružkov entropy-entropy flux pairs $\left(S_{c}(\cdot), Q_{c}(\cdot)\right)$ defined in (2.2). By (4.5) and (4.6),

$$
\begin{aligned}
& \iint_{\mathbb{R}_{\mathbb{R}_{+}}} S_{c}\left(u_{\Delta x}\right) \varphi_{t}+Q_{c}\left(u_{\Delta x}\right) \varphi_{x}+S_{c}^{\prime}\left(u_{\Delta x}\right) A\left(x, u_{\Delta x}\right) \varphi d x d t \\
& \quad+\int_{\mathbb{R}} S_{c}\left(u_{\Delta x}(x, 0)\right) \varphi(x, 0) d x \geq I_{2}(\varphi)+I_{3}(\varphi)+I_{4}(\varphi)+I_{5}(\varphi),
\end{aligned}
$$

for $\varphi \in C_{c}^{\infty}(\mathbb{R} \times[0, \infty))$. The term $I_{3}(\varphi)$ is nonnegative, while $I_{5}(\varphi)$ is zero if we choose $J$ sufficiently large. We are left with $I_{2}$ and $I_{4}$.

By convexity of $u \mapsto S_{c}(u)$ and (4.9),

$$
I_{2}(\varphi) \geq 0 \text {. }
$$

Regarding the term $I_{4}(\varphi)$, we use Lemma 4.7 and obtain

$$
\begin{aligned}
I_{4}(\varphi) & =\sum_{n, j} \int_{t^{n}}^{t^{n+1}}\left[Q_{c}\left(u_{j+1 / 2}^{n,-}\right)-Q_{c}\left(u_{j+1 / 2}^{n,+}\right)\right] \varphi\left(x_{j+1 / 2}, t\right) d t \\
& \geq-\sum_{n, j} \int_{t^{n}}^{t^{n+1}} \operatorname{sign}\left(\tilde{u}_{j+1 / 2}^{n}-c\right) \int_{x_{j}}^{x_{j+1}} A\left(x, u_{\Delta x}\left(x, t^{n}\right)\right) d x \varphi\left(x_{j+1 / 2}, t\right) d t
\end{aligned}
$$

where $\tilde{u}_{j+1 / 2}^{n}$ equals either $u_{j+1 / 2}^{n,-}$ or $u_{j+1 / 2}^{n,+}$ according to Lemma 4.7. Defining the piecewise constant functions $\tilde{u}_{\Delta x}, \hat{u}_{\Delta x}$ and $\varphi_{\Delta x}$ as

$$
\begin{aligned}
\tilde{u}_{\Delta x}(x, t) & =\sum_{j, n} \tilde{u}_{j+1 / 2}^{n} \mathbf{1}_{j+1 / 2}^{n}(x, t), \\
\hat{u}_{\Delta x}(x, t) & =\sum_{n} u^{n}(x) \mathbf{1}_{I^{n}}(t), \\
\varphi_{\Delta x}(x, t) & =\sum_{j} \varphi\left(x_{j+1 / 2}, t\right) \mathbf{1}_{I_{j+1 / 2}}(x),
\end{aligned}
$$

we have

$$
I_{4}(\varphi) \geq-\iint_{\Pi_{X, T}} \operatorname{sign}\left(\tilde{u}_{\Delta x}-c\right) A\left(x, \hat{u}_{\Delta x}\right) \varphi_{\Delta x} d x d t .
$$

From their definitions and using Lemma (4.5), it follows that,

$$
\begin{aligned}
\left\|\left(\tilde{u}_{\Delta x}-\hat{u}_{\Delta x}\right)\right\|_{L^{2}([0, T] \times(-X, X))} & \leq C \Delta t \Delta x \sum_{n=0}^{N-1} \sum_{j=-J}^{J}\left(u_{j+1 / 2}^{n,-}-u_{j+1 / 2}^{n,+}\right)^{2} \\
& +\left(u_{j}^{n}-u_{j+1 / 2}^{n,-}\right)^{2}+\left(u_{j+1}^{n}-u_{j+1 / 2}^{n,+}\right)^{2}=\mathcal{O}(\Delta t) .
\end{aligned}
$$

Similarly,

$$
\begin{aligned}
& \left\|\left(u_{\Delta x}-\hat{u}_{\Delta x}\right)\right\|_{L^{2}([0, T] \times(-X, X))} \\
& \quad \leq C \Delta t \Delta x \sum_{n=0}^{N-1} \sum_{j=-J}^{J}\left(u_{j}^{n}-u_{j+1 / 2}^{n,-}\right)^{2}+\left(u_{j+1}^{n}-u_{j+1 / 2}^{n,+}\right)^{2}=\mathcal{O}(\Delta t)
\end{aligned}
$$

Hence, it follows that

$$
\lim _{\Delta x \rightarrow 0} \tilde{u}_{\Delta x}=\lim _{\Delta x \rightarrow 0} \hat{u}_{\Delta x}=\lim _{\Delta x \rightarrow 0} u_{\Delta x}=u \quad \text { in } L_{\mathrm{loc}}^{1}\left(\mathbb{R} \times \mathbb{R}^{+}\right) .
$$


We also have that $\varphi_{\Delta x} \rightarrow \varphi$ for all $(x, t)$ as $\Delta x \rightarrow 0$. Hence, using Lemma 4.8,

$$
\lim _{\Delta x \rightarrow 0} I_{4}(\varphi) \geq-\iint_{\Pi_{X, T}} S^{\prime}(u) A(x, u) \varphi d x d t,
$$

for any $c \in \mathbb{R} \backslash \Theta$. Comparing this with the last term in the double integral in (4.12) and repeating the argument from [15] to extend the set of permissible $c$ 's to $\mathbb{R}$, we conclude that the limit $u$ is an entropy solution in the sense of Definition 2.3

Finally, we consider briefly the singular case (A.8), Combing the above chain of arguments with those found in [15] we can prove

Theorem 4.2. Suppose conditions (A.1) (A.4), (A.6), and (A.7) hold. Let $u_{\Delta x}$ be the approximate solutions generated by the well-balanced Godunov type scheme 3.2. Then there exists a limit function $u \in L^{\infty}\left(\mathbb{R} \times \mathbb{R}_{+}\right)$such that

$$
u_{\Delta x} \stackrel{\Delta x \rightarrow 0}{\longrightarrow} u \quad \text { in } L_{\mathrm{loc}}^{p}\left(\mathbb{R} \times \mathbb{R}_{+}\right) \text {for } p<\infty \text { and a.e. in } \mathbb{R} \times \mathbb{R}_{+} .
$$

Furthermore, $u$ is an entropy solution of (1.3) in the sense of Definition 2.4.

\section{Numerical EXPERIMENTS}

In this section we report several numerical experiments with our scheme (3.2), and compare it with the well-balanced scheme of [6] as well as with a standard centered source scheme. The well-balanced scheme of [6], which is formulated for the case (1.2), can be written as

$$
v_{j}^{n+1}=v_{j}^{n}-\lambda\left(F\left(v_{j}^{n}, v_{j+1 / 2}^{n,+}\right)-F\left(v_{j-1 / 2}^{n,-}, v_{j}^{n}\right)\right),
$$

where $F$ is a consistent and monotone numerical flux function. Moreover, $v_{j \pm 1 / 2}^{n, \pm}$ solve the algebraic equations

$$
D\left(v_{j \pm 1 / 2}^{n, \pm}\right)-z_{j}=D\left(v_{j \pm 1}^{n}\right)-z_{j \pm 1},
$$

where $D$ is defined by (4.3).

In our computations, we take $F$ in (5.1) to be the standard Godunov flux. We will refer to (3.2) as the AWBS scheme and (5.1) as the BPV scheme. The standard centered source scheme defined as

$$
w_{j}^{n+1}=w_{j}^{n}-\lambda\left(F\left(w_{j}^{n}, w_{j+1}^{n}\right)-F\left(w_{j-1}^{n}, w_{j}^{n}\right)-\frac{1}{2}\left(b\left(w_{j}^{n}\right)\left(z_{j+1}-z_{j}\right)\right)\right),
$$

where $F$ is any consistent and monotone numerical flux function. We will refer to the above scheme as the CS scheme.

Example 1. We start with an experiment that involves a nonlinear flux and a nontrivial bottom topography. Consider (1.1), (1.2) with

$$
f(u)=\frac{1}{2} u^{2}, \quad b(u)=u, \quad-z(x)= \begin{cases}\cos (\pi x), & 4.5<x<5.5, \\ 0, & \text { otherwise }\end{cases}
$$

In analogy with the shallow water equations, we refer to $z$ as the bottom topography. The topography $z$ is continuous in this case. This example is taken from [6]. We compute on the domain $[0,10]$ with initial data $u_{0} \equiv 0$ and boundary data $\left.u\right|_{x=0}=2$ to enforce the steady state. It is easy to see that the steady state is given by 


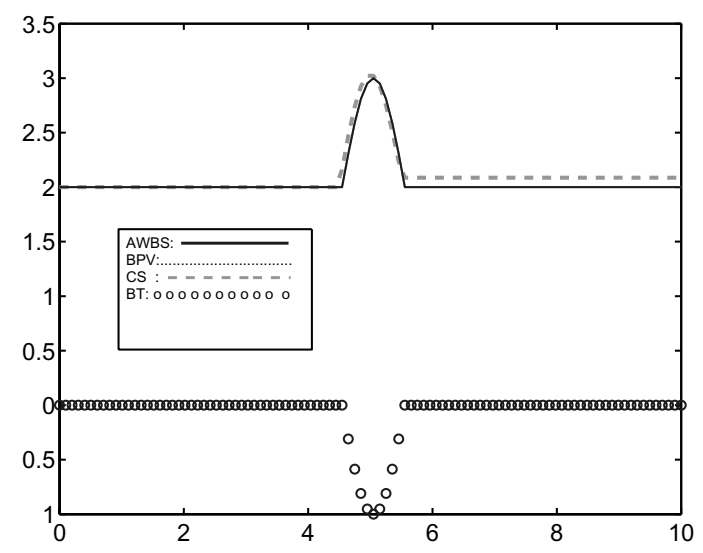

FiguRE 1. Discrete steady states computed by the three schemes for Example 1. The results of the AWBS and BPV schemes are indistinguishable. (BT refers to the bottom topography.)

$u(x)=2+z$. The steady state is reached once the shock front has passed the domain. The results at the steady state are shown in Figure 1 .

Note that the AWBS and BPV schemes resolve the steady state accurately even at this coarse mesh resolution, whereas the CS scheme does not resolve the steady state well. The errors at steady state are shown in Table 1 .

TABLE 1. Errors at the steady state for the CS, AWBS, and BPV schemes with $\Delta x=0.1$ in Example1.

\begin{tabular}{c|cc} 
& $L^{\infty}$ & $L^{1}$ \\
\hline CS & 0.1652 & 0.4824 \\
AWBS & $4.37 \times 10^{-14}$ & $2.22 \times 10^{-13}$ \\
BPV & $8.45 \times 10^{-14}$ & $2.26 \times 10^{-13}$
\end{tabular}

Next we focus on the transients and show the contour plots of the results with the AWBS and BPV schemes in Figure 2 ,

Observe that although both the AWBS and BPV schemes converge to the same steady state, their behavior in resolving transients is very different. In particular, when the right-moving shock is coming in from the boundary and has yet to reach the nontrivial dip in the bottom topography, the BPV scheme produces a traveling hump which appears to be nonphysical. On the other hand, as soon as the shock hits the dip, the BPV scheme closely resembles the solution computed by the AWBS scheme. To check whether the hump is a numerical artifact and disappears as $\Delta x \rightarrow 0$, we display the solutions computed by the BPV scheme $\Delta x=0.1,0.01$ in Figure 3. From Figure 3 it is clear that while one part of the hump seems to disappear in the limit, the part to the right seems to remain (and is in fact amplified) as the mesh is refined. Numerical experiments with the CS scheme (for a very fine mesh) show that there is no hump during the transient phase and the solutions agree with those computed by the AWBS scheme. This leads us to believe that the hump produced by the BPV scheme is not physical and is simply an artifact of the 

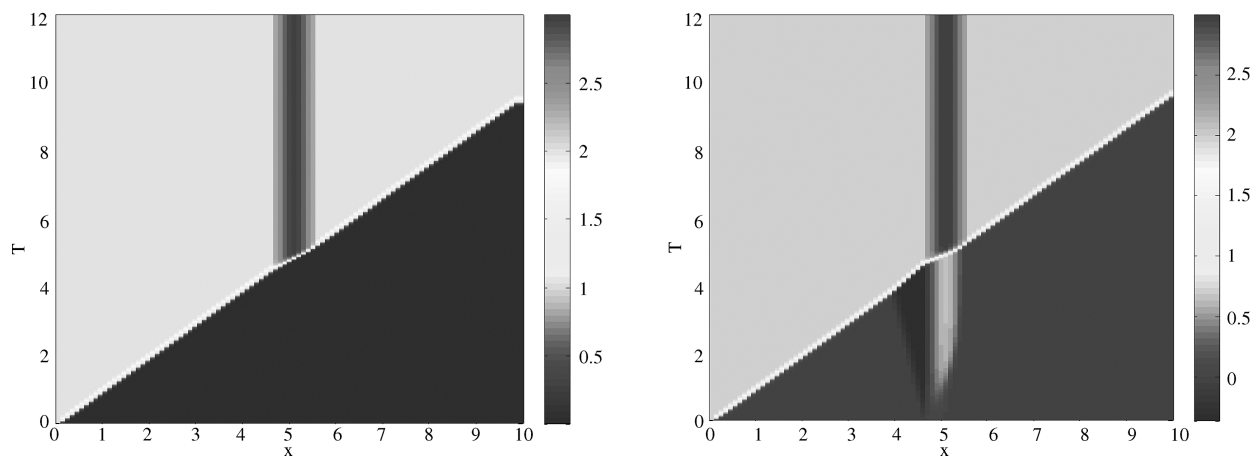

Figure 2. Contour plots in $(x, t)$ plane of the solutions computed by the AWBS and BPV schemes with $\Delta x=0.1$ in Example 1 .

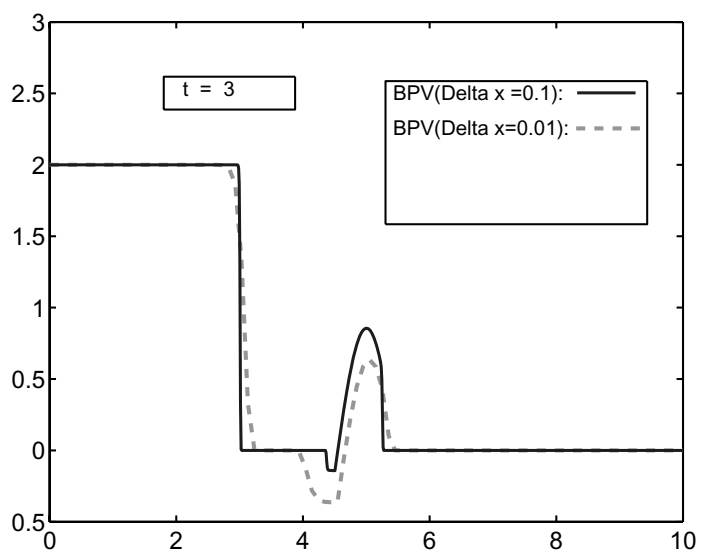

Figure 3. The two solutions computed by the BPV scheme with $\Delta x=0.1,0.01$ at time $t=3$ for Example 1.

scheme. Hence, the BPV scheme appears to converge to a different solution than the AWBS scheme in the transient phase of the flow.

Example 2. We consider the same problem as in Example1, except that we replace the bottom topography $z(x)$ by the discontinuous function

$$
-z(x)= \begin{cases}-\cos (\pi x), & 5<x<6, \\ 0, & \text { otherwise. }\end{cases}
$$

This topography is similar to the one given in [6] but with the opposite sign. The steady states are shown in Figure 4 and the transients are shown in Figure 5

In this case, both the steady states as well as the transient solutions given by the AWBS and BPV schemes are different. The steady state of the AWBS scheme is the entropy satisfying steady state as it satisfies the relation $u(x)=2+z(x)$, whereas the steady state given by the BPV does not satisfy this relation and hence is not the entropy solution. As in the previous experiment, the BPV scheme generates traveling waves almost instantaneously due to the effect of topography and converges to the the wrong steady state. Despite the discontinuities in the bottom 


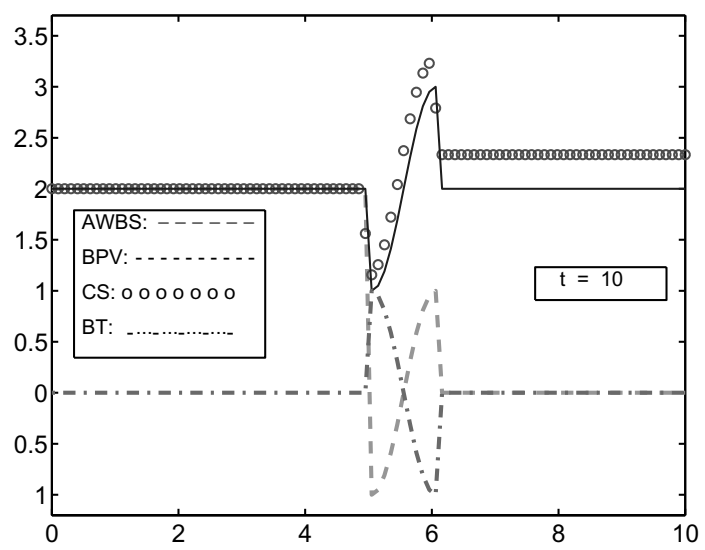

Figure 4 . The discrete steady states computed by the AWBS and BPV schemes with $\Delta x=0.1$ for Example 2.
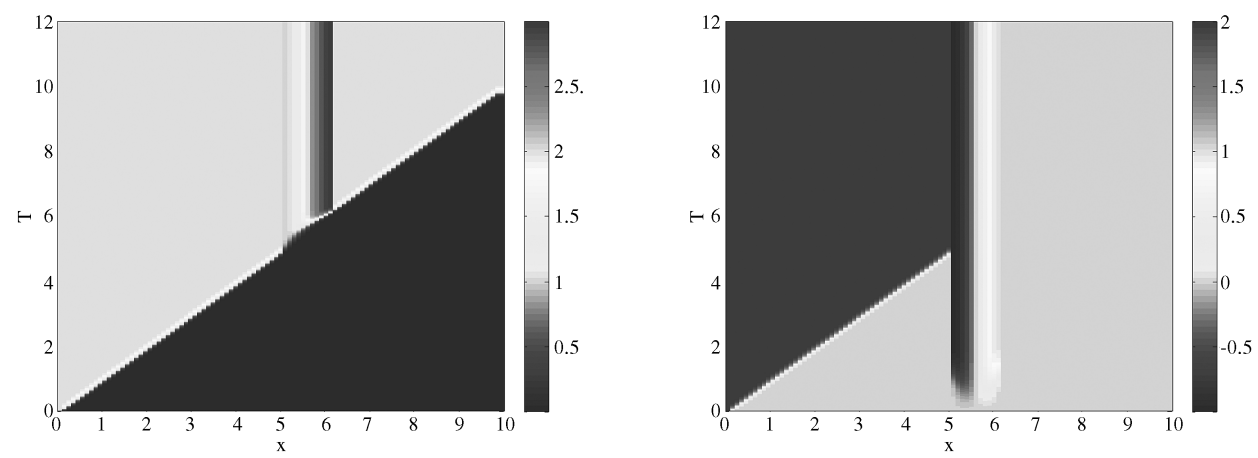

Figure 5. Contour plots in $(x, t)$ plane of the solutions computed by the AWBS and BPV schemes with $\Delta x=0.1$ in Example 2 .

topography $z(x)$, the AWBS scheme resolves the steady state almost to machine precision and correctly resolves the transient.

Example 3. In our third example, we consider the following sources and fluxes,

$$
u_{t}+\left(\frac{1}{2} u^{2}\right)_{x}=-z^{\prime}(x) u, \quad z(x)=-\cos (\pi x),
$$

with the initial data

$$
u(x, 0)=\cos (\pi x)+\frac{1}{10} \sin (4 \pi x) .
$$

We consider the above problem in the domain $[-1,1]$ with periodic boundary conditions and $\Delta x=0.02$. The exact steady state is given by

$$
\bar{u}(x)=\cos (\pi x) \text {. }
$$

Thus the initial data is a small periodic perturbation of the steady state and we expect the solution to converge to the steady state quickly. This problem is a prototype for quasi-steady problems. The exact solution consists of small amplitude waves which decay quickly to the steady state. We have computed the solution to this problem with the AWBS scheme and show the solutions in Figure 6 As shown 

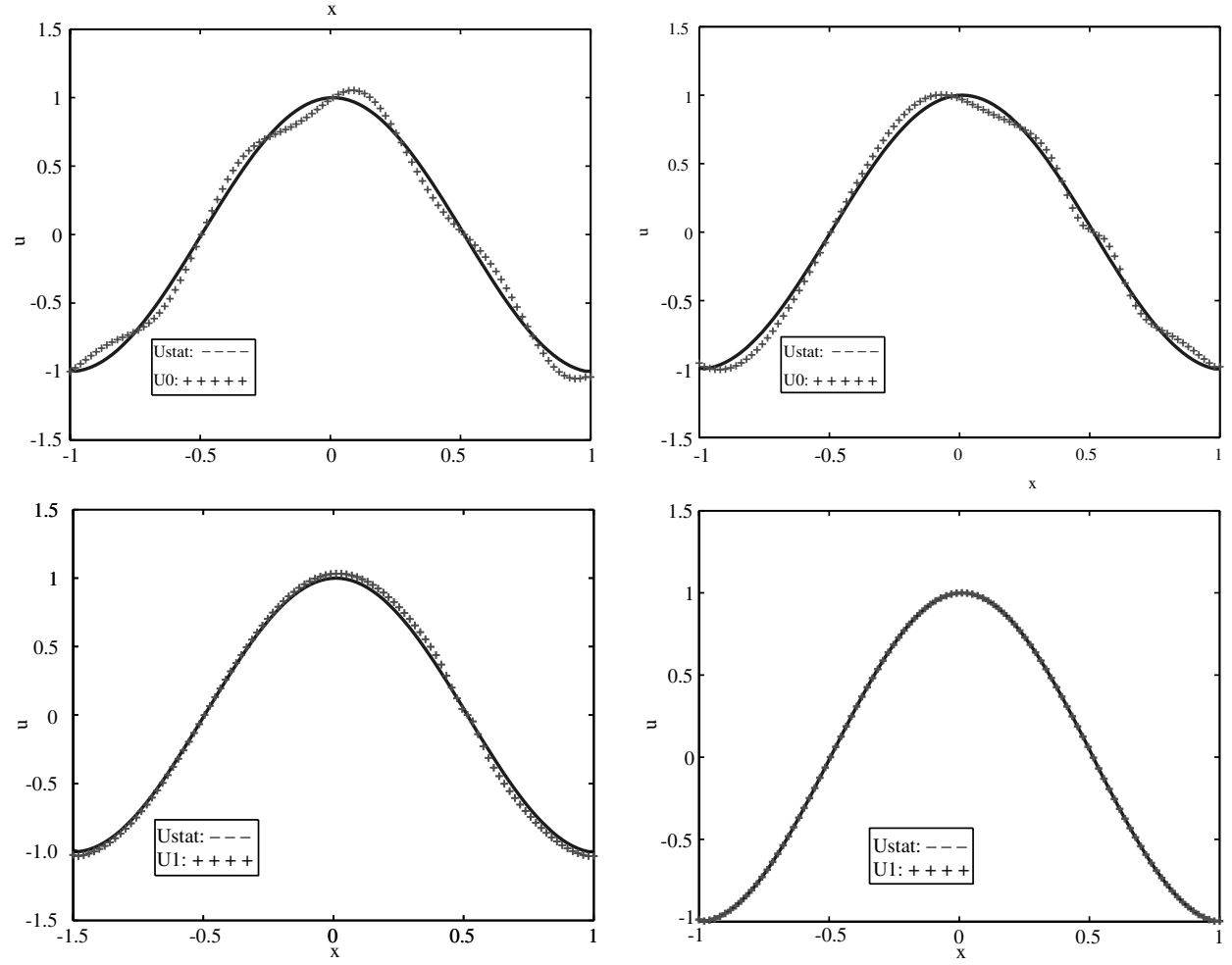

Figure 6. Approximations to solutions is experiment 3 with the AWBS scheme. Top left: The exact state and initial data. Top right: Exact state and solutions with AWBS scheme at $t=0.25$. Bottom left: Exact state and solutions with AWBS scheme at $t=$ 1. Bottom right: Exact steady state and solutions with AWBS scheme at $t=5$

in Figure 6, the initial conditions are a small periodic pertubation of the steady state. The AWBS scheme captures the small time transient behaviour quite well as shown in Figure 6. Furthermore, the solutions converge to the steady state by time $t=5$ and the AWBS scheme is able to capture this solution to machine precision. This example shows that the AWBS scheme is good at approximation of quasi-steady problems.

Example 4. In our fourth example we specify the relevant functions as follows:

$$
\begin{gathered}
f(u)=\frac{2}{3} u^{3}, \quad b(u)=u, \quad u_{0}(x)=1, \\
-z(x)= \begin{cases}\cos (\pi x), & 4.5<x<5.5, \\
0, & \text { otherwise }\end{cases}
\end{gathered}
$$

In this case, $D(u)=u^{2}$ and solutions to the steady state equation (4.3) may not exist or may be multi-valued. Extra care is required to define the BPV scheme (see [6] for details), whereas the AWBS scheme is well-defined. In fact, it is very easy to 

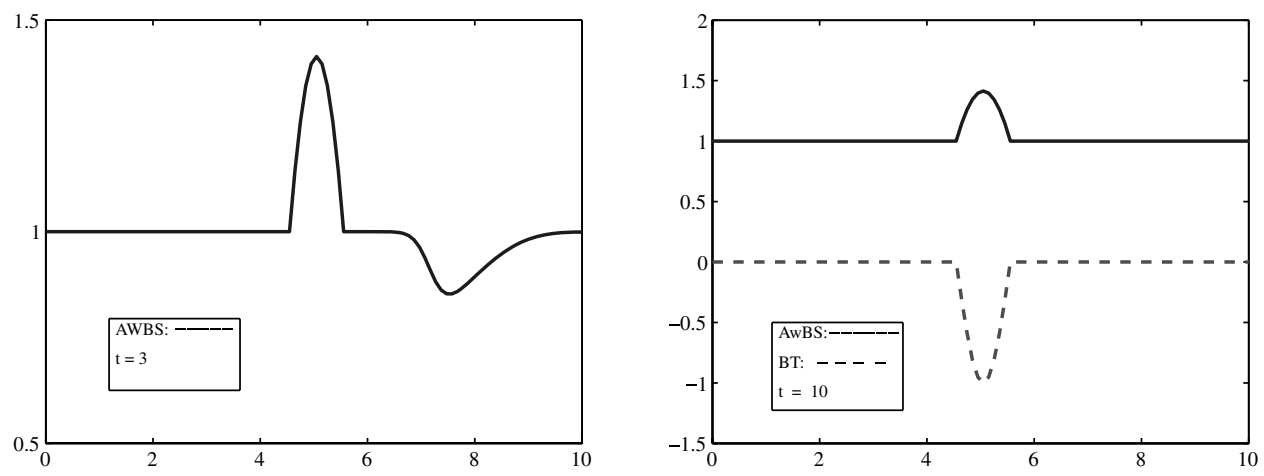

Figure 7. Solutions computed by the AWBS scheme at $t=3,10$ with $\Delta x=0.1$ for Example 3, (BT refers to bottom topography.)

implement as the flux function is monotone in this case. The transient and steady state solutions computed by the AWBS scheme are shown in Figure 7.

We observe that the resolution of the steady state, which in this example is given by $\bar{u}(x)=\sqrt{1+z(x)}$, is resolved to almost machine precision. Additionally, the transients are resolved very well on this coarse mesh as well.

Example 5. In our final example we specify the data as follows:

$$
\begin{gathered}
f(u)=\frac{1}{2} u^{2}, \quad A(x, u)=\sin \left(2 \pi x u^{2}\right), \\
u_{0}(x)=0, \quad u(0, t)=1 .
\end{gathered}
$$

In this example the structure of the source term is very general and well-balancing based on the nonlinear transformation $D$ and (4.3) is not possible, whereas the AWBS scheme is well defined. We have computed solutions on the domain $[0,5]$ and determined the steady state by solving the ODE

$$
\left(u^{2}\right)_{x}=A(x, u)
$$

by a high-order Runge-Kutta method. The steady state and transient solutions are shown in Figure 8

The steady state is resolved quite well with the AWBS scheme. It is not immediately clear how to modify other well-balanced schemes (like the BPV scheme) so that they can be applied to the present problem. We remark that in this particular example it was easy to determine the solution of the ODE for the steady state as it turned out to be a smooth function. In general, however, it may not be possible to obtain entropy satisfying steady states from ODE solvers without first building the entropy condition into the solver, whereas the AWBS scheme by construction automatically captures the entropy solution. 

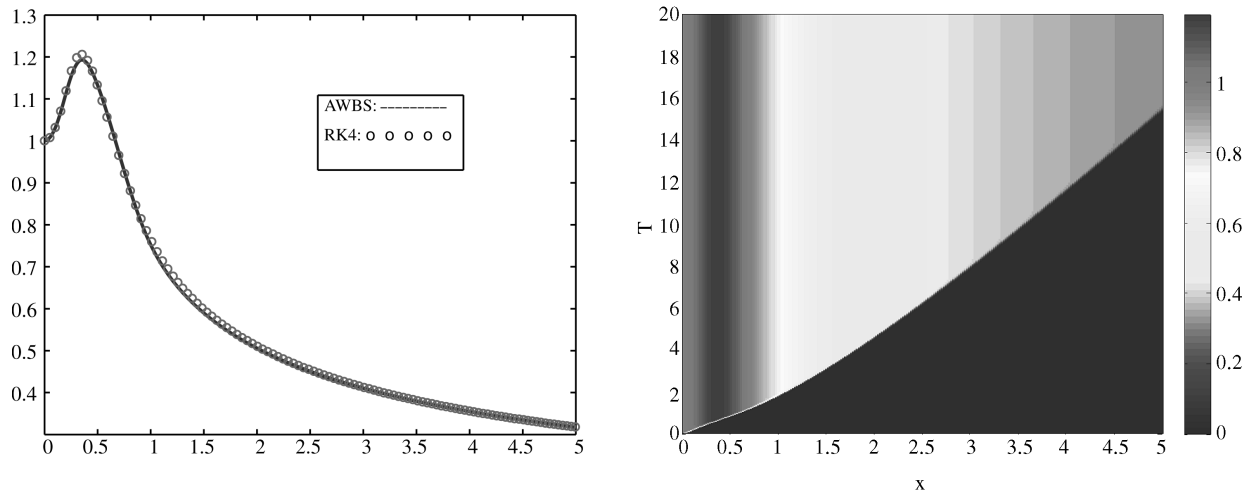

Figure 8 . Solutions computed by the AWBS scheme at $t=20$ with $\Delta x=0.02$ for Example 3 .

\section{REFERENCES}

[1] Adimurthi, J. Jaffre and G. D.Veerappa Gowda. Godunov type methods for scalar conservation laws with flux function discontinuous in the space variable. SIAM J. Numer. Anal., 42 (1):179-208, 2004. MR2051062 (2005c:65066)

[2] Adimurthi, Siddhartha Mishra and G. D.Veerappa Gowda. Optimal entropy solutions for conservation laws with discontinuous flux functions. J. Hyperbolic Differ. Equ., 2 (4):1-56, 2005. MR2195983 (2007g:35144)

[3] Adimurthi, S. Mishra, and G. D. V. Gowda. Existence and stability of entropy solutions for a conservation law with discontinuous non-convex fluxes. Netw. Heterog. Media, 2(1):127-157 (electronic), 2007. MR 2291815

[4] F. Bachmann and J. Vovelle. Existence and uniqueness of entropy solution of scalar conservation laws with a flux function involving discontinuous coefficients. Comm. Partial Differential Equations, 31(1-3):371-395, 2006. MR2209759 (2008b:35170)

[5] D. S. Bale, R. J. Leveque, S. Mitran, and J. A. Rossmanith. A wave propagation method for conservation laws and balance laws with spatially varying flux functions. SIAM J. Sci. Comput., 24(3):955-978 (electronic), 2002. MR1950520 (2004a:65098)

[6] R. Botchorishvili, B. Perthame, and A. Vasseur. Equilibrium schemes for scalar conservation laws with stiff sources. Math. Comp., 72(241):131-157 (electronic), 2003. MR1933816 (2003h:65104)

[7] G.-Q. Chen. Compactness methods and nonlinear hyperbolic conservation laws. In Some current topics on nonlinear conservation laws, volume 15 of AMS/IP Stud. Adv. Math., pages 33-75. Amer. Math. Soc., Providence, RI, 2000. MR.1767623 (2001f:35249)

[8] X. Ding, G.-Q. Chen and P. Luo. Convergence of the Lax-Friedrichs scheme for isentropic gas dynamics. Acta. Math. Sci. 5, 483-500, 1985 (in English), 7, 467-480, 1987 (in Chinese). MR.943646 (90b:76001)

[9] R. J. DiPerna. Convergence of approximate solutions to conservation laws. Arch. Rational Mech. Anal., 82(1):27-70, 1983. MR684413 (84k:35091)

[10] T. Gimse and N. H. Risebro. Solution of the Cauchy problem for a conservation law with a discontinuous flux function. SIAM Journal on Mathematical Analysis, 23(3):635-648, 1992. MR.1158825 (93e:35070)

[11] L. Gosse and A.-Y. Leroux. Un schéma-équilibre adapté aux lois de conservation scalaires non-homogènes. C. R. Acad. Sci. Paris Sér. I Math., 323(5):543-546, 1996. MR:1408992 (97i:35112)

[12] L. Gosse. Localization effects and measure source terms in numerical schemes for balance laws. Math. Comp., 71(238):553-582 (electronic), 2002. MR1885615 (2003e:65147)

[13] J. M. Greenberg and A. Y. Leroux. A well-balanced scheme for the numerical processing of source terms in hyperbolic equations. SIAM J. Numer. Anal., 33(1):1-16, 1996. MR1377240 (97c:65144) 
[14] K. H. Karlsen, N. H. Risebro, and J. D. Towers. $L^{1}$ stability for entropy solutions of nonlinear degenerate parabolic convection-diffusion equations with discontinuous coefficients. $S k r . K$. Nor. Vidensk. Selsk., (3):1-49, 2003. MR2024741 (2004j:35149)

[15] K. H. Karlsen, N. H. Risebro, and J. D. Towers. Front tracking for scalar balance equations. J. Hyperbolic Differ. Equ., 1(1):115-148, 2004. MR2052473(2005d:35156)

[16] K. H. Karlsen and J. D. Towers. Convergence of the Lax-Friedrichs scheme and stability for conservation laws with a discontinous space-time dependent flux. Chinese Ann. Math. Ser. $B, 25(3): 287-318,2004$. MR2086124(2005h:65145)

[17] K. H. Karlsen, S. Mishra, and N. H. Risebro. Convergence of finite volume schemes for triangular systems of conservation laws. Submitted, 2006.

[18] C. Klingenberg and N. H. Risebro. Convex conservation laws with discontinuous coefficients. Existence, uniqueness and asymptotic behavior. Comm. Partial Differential Equations, 20(11-12):1959-1990, 1995. MR.1361727 (96i:35082)

[19] R. J. LeVeque. Finite volume methods for hyperbolic problems. Cambridge Texts in Applied Mathematics. Cambridge University Press, Cambridge, 2002. MR.1925043 (2003h:65001)

[20] Y. Lu. Hyperbolic conservation laws and the compensated compactness method, volume 128 of Chapman $\&$ Hall/CRC Monographs and Surveys in Pure and Applied Mathematics. Chapman \& Hall/CRC, Boca Raton, FL, 2003. MR1936672 (2004b:35223)

[21] S. Mishra. Convergence of upwind finite difference schemes for a scalar conservation law with indefinite discontinuities in the flux function. SIAM J. Numer. Anal., 43(2):559-577 (electronic), 2005. MR2177880 (2006g:65131)

[22] S. Mishra. Analysis and Numerical approximation of conservation laws with discontinuous coefficients Ph.D. Thesis, Indian Institute of Science, Bangalore, 2005.

[23] F. Murat. L'injection du cône positif de $H^{-1}$ dans $W^{-1, q}$ est compacte pour tout $q<2$. J. Math. Pures Appl. (9), 60(3):309-322, 1981. MR633007(83b:46045)

[24] A. Noussair. Riemann problem with nonlinear resonance effects and well-balanced Godunov scheme for shallow fluid flow past an obstacle. SIAM J. Numer. Anal., 39(1):52-72 (electronic), 2001. MR $1860716(2002 \mathrm{~h}: 35182)$

[25] L. Tartar. Compensated compactness and applications to partial differential equations. In Nonlinear analysis and mechanics: Heriot-Watt Symposium, Vol. IV, pages 136-212. Pitman, Boston, Mass., 1979. MR584398 (81m:35014)

[26] L. Tartar. The compensated compactness method applied to systems of conservation laws. In Systems of nonlinear partial differential equations (Oxford, 1982), volume 111 of NATO Adv. Sci. Inst. Ser. C Math. Phys. Sci., pages 263-285. Reidel, Dordrecht, 1983. MR725524 (85e:35079)

[27] J. D. Towers. Convergence of a difference scheme for conservation laws with a discontinuous flux. SIAM J. Numer. Anal., 38(2):681-698 (electronic), 2000. MR1770068 (2001f:65098)

[28] J. D. Towers. A difference scheme for conservation laws with a discontinuous flux: The nonconvex case. SIAM J. Numer. Anal., 39(4):1197-1218 (electronic), 2001. MR1870839 (2002k:65131)

Centre of Mathematics for Applications (CMA), University of Oslo, P.O. Box 1053, BLINDERn, N-0316 OsLO, NorWAY

E-mail address: kennethk@math.uio.no

Centre of Mathematics for Applications (CMA), University of Oslo, P.O. Box 1053, Blindern, N-0316 Oslo, Norway

E-mail address: siddharm@cma.uio.no

Centre of Mathematics for Applications (CMA), University of Oslo, P.O. Box 1053, BLINDERN, N-0316 OSLO, NORWAY

E-mail address: nilshr@math.uio.no 